\title{
Téoros
}

Revue de recherche en tourisme

\section{Autopsie de la restauration au Québec}

\section{Jean Stafford et Bruno Sarrasin}

Volume 19, numéro 2, été 2000

URI : https://id.erudit.org/iderudit/1071969ar

DOI : https://doi.org/10.7202/1071969ar

Aller au sommaire du numéro

Éditeur(s)

Université du Québec à Montréal

ISSN

0712-8657 (imprimé)

1923-2705 (numérique)

Découvrir la revue

Citer cette note

Stafford, J. \& Sarrasin, B. (2000). Autopsie de la restauration au Québec. Téoros, 19(2), 61-63. https://doi.org/10.7202/1071969ar d'utilisation que vous pouvez consulter en ligne.

https://apropos.erudit.org/fr/usagers/politique-dutilisation/ 


\section{Autopsie de la restauration Au Québec}

\section{Jean Stafford et Bruno Sarrasin}

Cette chronique porte sur la conjoncture touristique. Elle propose un portrait simplifié de la situation du tourisme à partir d'une clientèle précise et des données disponibles. Les notions usuelles de l'analyse prévisionnelle sont utilisées : la tendance (passée, présente et future), la saisonnalité, le cycle et les mouvements irréguliers et des prévisions sont faites pour les cinq prochaines années.

Depuis les années 1990, on peut constater une augmentation constante des dépenses en restauration au Québec et au Canada et, plus récemment, cette progression tend à s'intensifier ; en effet, selon Statistique Canada, « De 1989 à 1998, les dépenses personnelles en repas pris à l'extérieur de la maison se sont accrues de 42,2\%, alors que les sommes consacrées à l'achat d'aliments et de boissons dans les supermarchés, les épiceries et autres magasins d'alimentation ont augmenté de $30,3 \%{ }^{1} »$. Par ailleurs, les dépenses en restauration dépendent des revenus et de la taille des ménages.

En 1998, les dépenses en restauration regroupaient près de $20 \%$ des dépenses touristiques $(1,125 \text { milliard } \$)^{2}$ effectuées au Québec ; il s'agit du troisième secteur en importance après les transports et l'hébergement. De plus, le domaine de la restauration représente $35 \%$ de 1'emploi en tourisme au Québec ${ }^{3}$.

\section{L'ÉVOLUTION DES RECETTES DE LA RESTAURATION AU QUÉBEC SELON LE TYPE D'ÉTABLISSEMENTT}

Si on réfère aux taux d'accroissement annuels moyens des recettes de la restauration au Québec selon le type d'établissements ${ }^{4}$, on constate que la période 1983-1987 a été assez faste pour les restaurants licenciés et non-licenciés et pour les établissements qui vendent des aliments à emporter, alors que l'accroissement a été très faible pour les traiteurs et les tavernes.

La période suivante, 1988-1992, a été plutôt néfaste pour la plupart des entreprises : on assiste à une baisse des recettes pour les établissements non-licenciés, les traiteurs et les tavernes. Pendant ce temps, les établissements licenciés et les firmes qui vendent des aliments à emporter connaissent respectivement une progression de $2,5 \%$ et de $1,9 \%$ par année.

Au cours de la troisième période, 19931997 , on peut constater un retournement de la situation pour les traiteurs ( $5 \%$ d'augmentation des recettes). Les entreprises qui vendent des repas à emporter ont connu une croissance spectaculaire ( $7,2 \%$ d'augmentation annuelle) et les restaurants licenciés ont eu une croissance de 3,8\% annuellement. Pendant cette même période, les restaurants non-licenciés étaient en perte de vitesse, avec une diminution importante des recettes de $-5,4 \%$ par année ; les tavernes ont aussi été touchées par cette situation avec une décroissance annuelle des recettes de $-3,3 \%$.

La lecture de ces données montre que les établissements les plus performants au cours des quinze dernières années sont, d'une part, les entreprises qui vendent des aliments à emporter (croissance annuelle de $4,7 \%$ ) et, d'autre part, les restaurants licenciés (avec $4,1 \%$ ) et les traiteurs ( $4 \%$ ). Les derniers de classe sont les restaurants non-licenciés et les tavernes (baisse annuelle respective de $-1,3 \%$ et de $-1,1 \%$ ). Ces données traduisent certainement des changements dans les goûts des consommateurs.

\section{L'ÉVOLUTION INDICIELLE DES RECETTES DE LA RESTAURATION}

L'évolution indicielle permet de comparer la progression des différents établissements sans tenir compte du nombre de ceux-ci dans chacune des catégories. Les indices des recettes totales montrent les crises majeures qui ont touché de front l'ensemble du domaine de la restauration : la première crise en 1982, 1983 et 1984 et la deuxième en 1991, 1992 et 1993.

Le graphique 1 permet de suivre l'évolution de chacun des types d'établissements : les recettes des restaurants licenciés ont une évolution croissante pour l'ensemble de la période ; les restaurants non-licenciés connaissent une baisse graduelle de leurs recettes depuis 1990 ; les établissements qui vendent des repas à emporter ont des indices des recettes en dessous de la valeur 100 , ce qui indique que ce type de restauration n'a jamais réussi à rejoindre le niveau des recettes de 1981.

Les traiteurs ont connu, en matière d'évolution des indices des recettes, un long passage à vide de 1981 à 1987 et, par la suite, de 1988 à 1990, une croissance plus élevée. Les années récentes montrent une 
grande stabilité des indices de 1992 à 1996 et une brusque remontée en 1997 (avec 141). Enfin, les tavernes présentent une évolution en dents de scie de 1981 à 1989 et une baisse lente des valeurs des indices des recettes de 1991 à 1997.

\section{LES PARTS DE MARCHÉ DES RECETTES DE LA RESTAURATION SELON LE TYPE D'ÉTABLISSEMENTS}

Le graphique 2 présente les parts de marché, en pourcentage, selon le type d'établissements, pour les années 1981 à 1997. Les restaurants licenciés et les restaurants non-licenciés accaparent, à eux deux, en moyenne et pour l'ensemble de la période étudiée, près de $73 \%$ des recettes. Cette moyenne cache une profonde inégalité entre ces deux types d'établissements. En 1981, les parts de marché étaient de $39 \%$ pour les restaurants licenciés et de $28 \%$ pour les restaurants nonlicenciés et, en 1997, les restaurants licenciés possédaient près de $54 \%$ du marché et les non-licenciés seulement $19 \%$.

Les parts de marché des tavernes arrivent en troisième place ; les parts de marché de cette catégorie d'établissements oscillent de $8 \%$ à $14 \%$ de 1981 à 1997, la moyenne étant de 11,1\%. En 1997, les tavernes détenaient $8 \%$ de l'ensemble des recettes de la restauration ; c'est un marché en lent déclin depuis 1990.

Les entreprises qui vendent des repas à emporter ont une faible part du marché de la restauration. La moyenne pour la période considérée est de 8,8\%. Dans l'ensemble, les parts de marché de ce type d'établissements sont assez stables depuis 1989. Les parts de marché des traiteurs à ce chapitre varient de $6 \%$ à $8 \%$ de 1981 à 1987 , la moyenne étant de $6,9 \%$ pour la période. Ce type de restauration montre donc une grande stabilité (ou stagnation?).

\section{LES FLUCTUATIONS ${ }^{5}$ DES SÉRIES TEMPORELLES DES RECETTES DE LA RESTAURATION}

Les fluctuations des séries temporelles des recettes de la restauration sont, dans l'ensemble (et c'est une bonne chose), plus faibles que celles du domaine de l'hébergement.

\section{GRAPHIQUE 1 :}

L'évolution indicielle des recettes de la restauration au Québec, selon les établissements, base $100=1981$

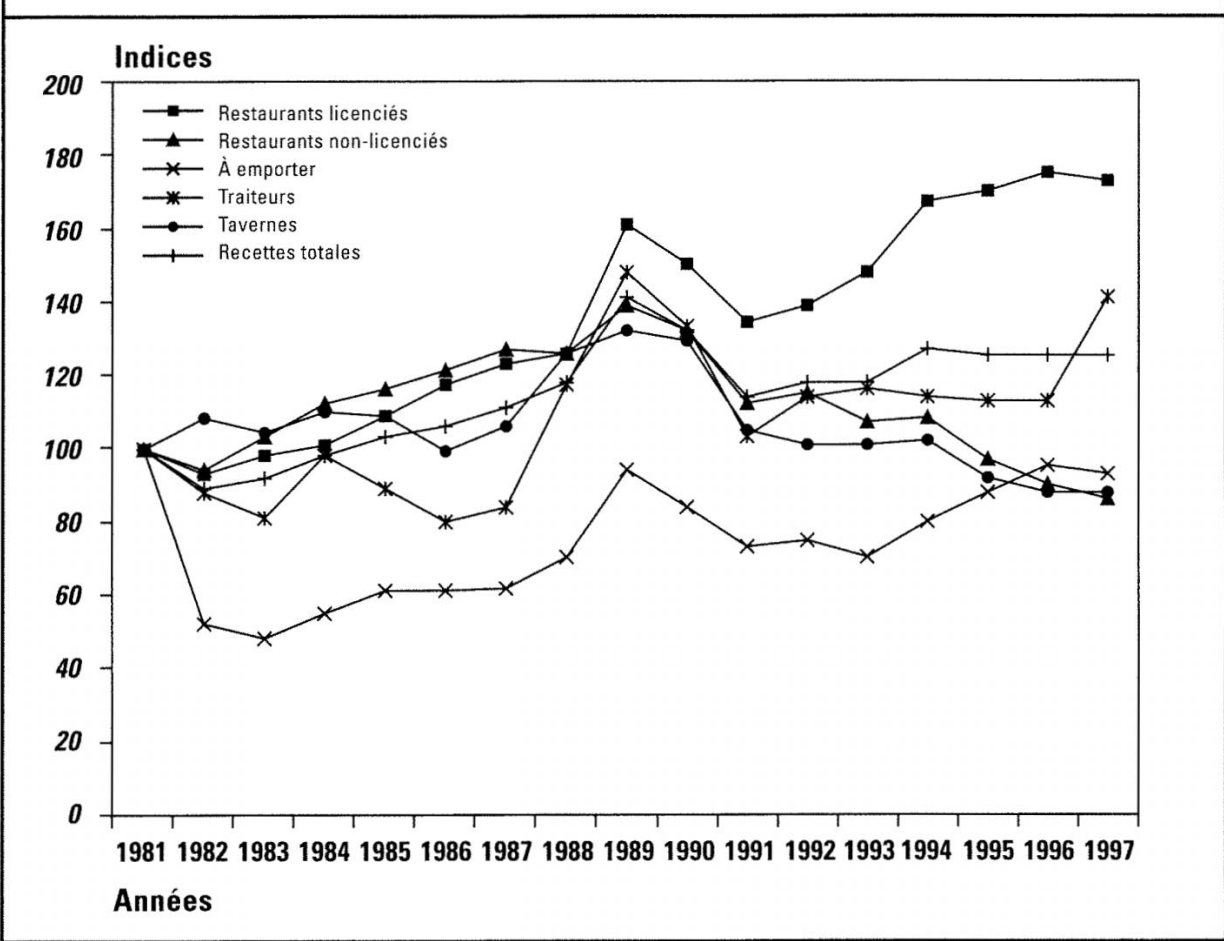

GRAPHIQUE 2 :

Les parts du marché des recettes de la restauration au Québec, de 1981 à 1997 en pourcentage

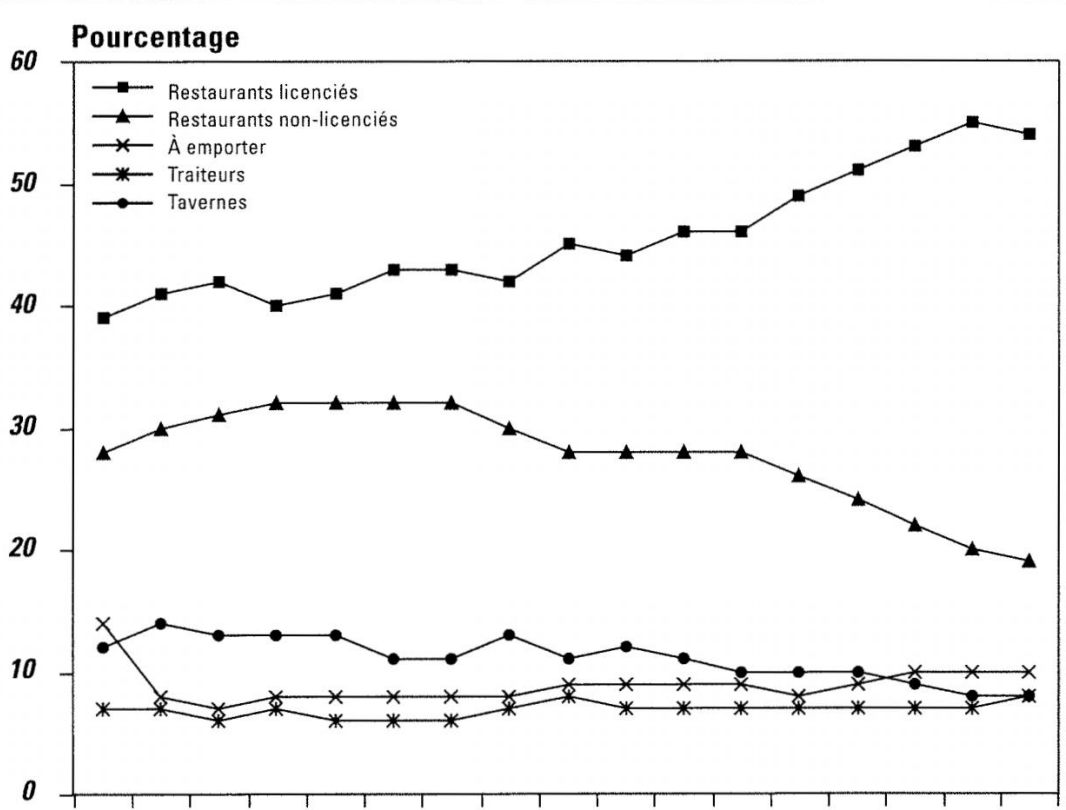

19811982198319841985198619871988198919901991199219931994199519961997

Années 
Le graphique 3 donne les coefficients saisonniers des recettes de la restauration selon le type d'établissements, pour la période 1993-1997. Pendant le premier trimestre de l' année, les coefficients saisonniers des restaurants licenciés, nonlicenciés et des entreprises qui vendent des repas à emporter sont presque semblables. Les traiteurs et les tavernes semblent avoir une activité plus élevée que les autres types d'établissements pendant ce trimestre.

Pendant le deuxième trimestre, les entreprises qui vendent des repas à emporter se détachent du peloton ; on peut voir (graphique 3) que le coefficient saisonnier est de 115 pour ce type d'établissements. Les restaurants non-licenciés ont un coefficient saisonnier un peu plus élevé que celui des trois autres types.

Au troisième trimestre, ce sont les restaurants licenciés et non-licenciés qui ont les coefficients saisonniers les plus élevés. On constate une baisse sensible des coefficients chez les traiteurs (sous la barre de 100).

Au quatrième trimestre, les traiteurs prennent la tête (avec un coefficient saisonnier de 106), suivis des tavernes (coefficient saisonnier de 102). Les autres établissements sont sous la ligne fatidique de 100 , ce qui dénote une activité normale ou sous la normale !

Pour l'ensemble des établissements, les phénomènes irréguliers sont faibles et négligeables (les écarts types varient de $1 \%$ à $5 \%$ ). Les phénomènes cycliques ne sont pas inquiétants (contrairement à beaucoup d'autres secteurs du tourisme). Les écarts types des coefficients cycliques oscillent de $7 \%$ à $12 \%$; les établissements qui sont les plus sensibles aux cycles économiques sont les entreprises qui vendent des repas à emporter et les traiteurs.

\section{LES TENDANCES ${ }^{6}$ DANS L'ÉVOLUTION FUTURE DES RECETTES DE LA RESTAURATION AU QUÉBEC}

Les recettes totales de la restauration devraient augmenter faiblement, toutes choses étant égales par ailleurs, au cours des années 2000 à 2004 (croissance de $1 \%$ à $2 \%$ par année pendant cette période).

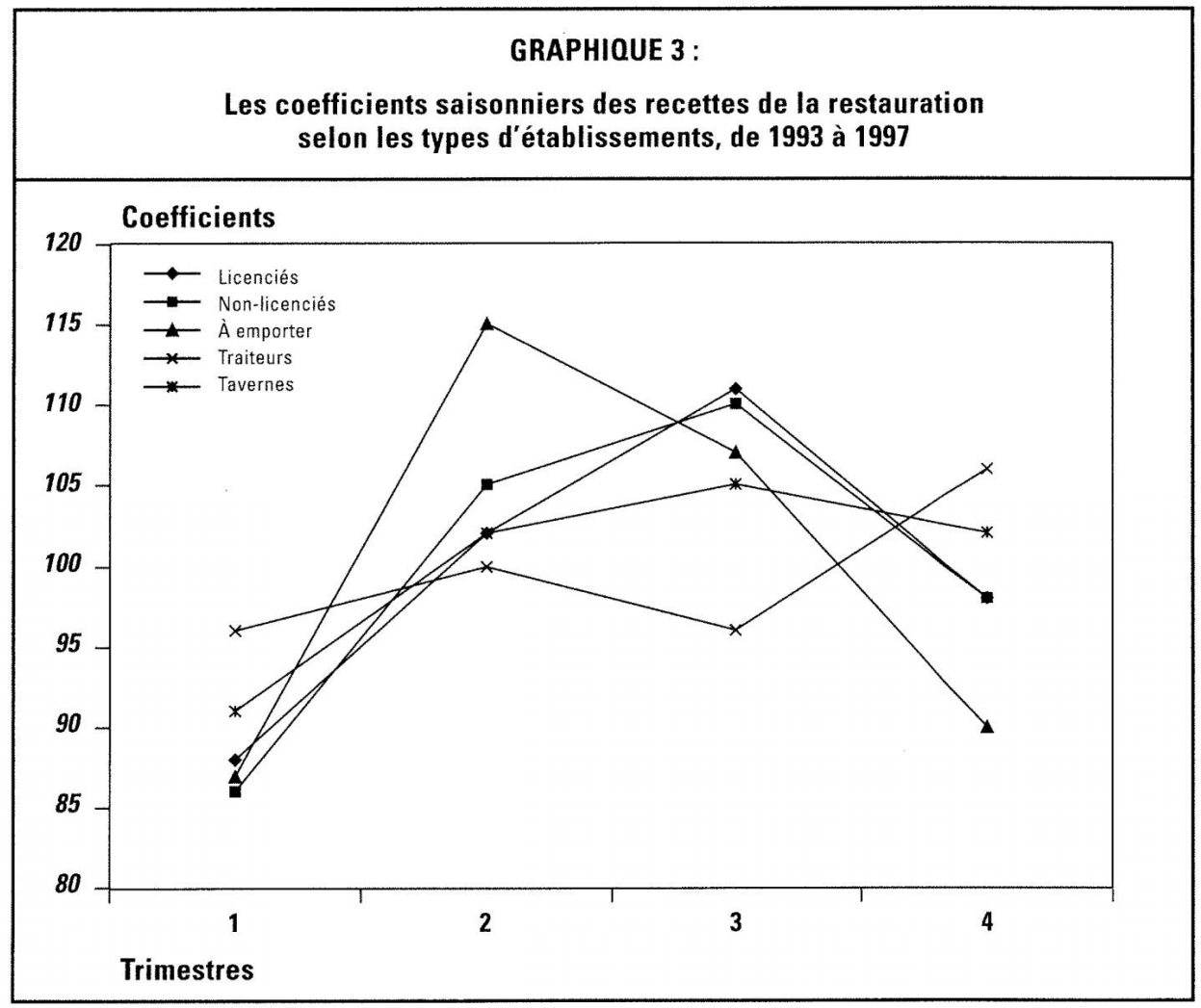

Les recettes de la restauration selon le type d'établissements, au cours de la période 2000 à 2004, devraient évoluer de la façon suivante :

- les restaurants licenciés auront une croissance annuelle de $5 \%$;

- les restaurants non-licenciés connaîtront une baisse annuelle de $-3 \%$ à $-4 \%$ pendant cette période ;

- les entreprises qui vendent des repas à emporter et les traiteurs auront un accroissement annuel de leurs recettes de $4,5 \%$ à $5,5 \%$;

- enfin, les tavernes verront une baisse graduelle de leurs recettes de $-6 \%$ dans les cinq prochaines années.

Dans l'ensemble, l'évolution des recettes de la restauration au Québec est très contrastée ; le type de restauration a un effet direct sur les recettes attendues. Les restaurants non-licenciés et les tavernes sont nettement en perte de vitesse, alors que les restaurants licenciés détiennent la grosse part du marché. Les entreprises qui vendent des repas à emporter et les traiteurs, pour leur part, se maintiennent à flot, mais semblent plafonner en matière de parts de marché depuis une dizaine d'années. Il y a sûrement là un problème à approfondir.
Jean Stafford est professeur et Bruno Sarrasin est chargé de cours, tous deux au Département d'études urbaines et touristiques de l'UQAM.

\section{NOTES}

1 Le Quotidien, Statistique Canada, 14 avril 2000.

2 Tourisme Québec (2000), Le tourisme au Québec en 1998. Une réalité économique importante, Québec, p. 21.

3 Ibid, p. 42.

4 Source : nos calculs à partir des données de Statistique Canada : «Statistiques des restaurants, traiteurs et tavernes ».

5 Les calculs des fluctuations sont faits à partir de la méthode de « décomposition des séries chronologiques» du logiciel SPSS sous Windows.

6 Il s'agit ici de simples projections des tendances passées des séries chronologiques de la restauration: ces tendances tiennent compte des différents mouvements analysés : mouvements tendanciels, saisonniers, cycliques et irréguliers. Il faut considérer ces prévisions comme des hypothèses sur le développement possible de ce secteur du tourisme. 